\title{
Left paranasal hemophilic pseudotumor in a 5-year-old boy: A case report
}

\author{
Saleh Yuguda, ${ }^{1}$ Ahmed Iya Girei, ${ }^{1}$ Rufai Abdu Dachi, ${ }^{2}$ Sani Adamu ${ }^{3}$ \\ ${ }^{1}$ Department of Hematology and Blood Transfusion, Gombe State University/Federal Teaching Hospital Gombe, \\ Gombe State; ${ }^{2}$ Department of Hematology and Blood Transfusion, Abubakar Tafawa Balewa University \\ Teaching Hospital/Abubakar Tafawa Balewa University Bauchi, Bauchi State; ${ }^{3}$ Department of Surgery, \\ Pediatric Surgery Unit, Gombe State University/Federal Teaching Hospital Gombe, Gombe State, Nigeria
}

\begin{abstract}
Hemophilic pseudotumors are rare complications of hemophilia that are seen in $1-2 \%$ of patients commonly affecting patients with severe disease. Hemophilic pseudotumors occur as a result of recurrent poorly managed or untreated bleeding either in the soft tissue or bone. We report a 5-year-old boy with a previously undiagnosed hemophilia A who developed left paranasal swelling following a fall from a height. He was diagnosed with hemophilic pseudotumor and successfully managed conservatively with factor VIII replacement.
\end{abstract}

\section{Introduction}

Hemophilic pseudotumors are rare complications of Hemophilia A and B. They are estimated to occur in about $1-2 \%$ of patients. ${ }^{1}$ They are commoner among patients with severe Hemophilia and usually occur in the long bones, however soft tissues and cartilages can also be involved. Hemophilic pseudotumors, structurally composed of bleeds resulting from repeated hemorrhage in to soft tissues, subperiosteum or site of bone fracture with inadequate resorption of the extravasated blood in patients with hemophilia. Involvement of paranasal region are extremely rare and usually result from repeated untreated or poorly treated bleeding following trauma. ${ }^{1}$ We report a case of left paranasal pseudotumor in a 5 -year-old boy who was managed conservatively with factor VIII replacement.

\section{Case Report}

He was a 5-year-old boy who presented to Federal Teaching Transfusion, College of Medical Sciences, Gombe State University, Gombe, Gombe State, Nigeria.

Tel.: +2348097470075 .

E-mail: yugudas@gmail.com

Key words: Hemophilia, pseudotumor, paranasal.

Acknowledgements: the authors want to acknowledge the support of the Humanitarian Aids Program of the World Federation of Hemophilia in providing us with free factor concentrates for use through the Hemophilia Foundation of Nigeria.

Contributions: SY: participated in the management of the patient, design, writing, review and final approval of the paper; AIG: participated in the design and review of the paper; RAD: participated in the management of the patient, design, writing review and final approval of the paper; SA: participated in the management of the patient, design, review and final approval of the paper.

Conflict of interest: the authors declare no potential conflict of interest.

Funding: none.

Received for publication: 30 June 2019.

Revision received: 7 January 2020.

Accepted for publication: 7 January 2020.

This work is licensed under a Creative Commons Attribution NonCommercial 4.0 License (CC BY-NC 4.0).

${ }^{\circ}$ Copyright: the Author(s), 2019

Licensee PAGEPress, Italy

Annals of African Medical Research 2019; 2:79

doi:10.4081/aamr.2019.79
Hospital (FTH) Gombe with left paranasal swelling following a fall from a height which he sustained about two (2) weeks prior to presentation. He was said to have bled from the nose for about 5 days before referral to us. There was a positive history of severe post-circumcision bleeding for which he was transfused with seven (7) units of blood at a General Hospital. No associated history of fever and no swelling in any other part of the body. The swelling did not interfere with breathing or sight. He was the first child from his mother with two other siblings who were both alive and well with no history of bleeding. There was no history suggestive of hemophilia from the maternal uncles, male children of mother's sisters or male children of grandaunts. On examination, he was found to have a swelling in the left para-nasal region measuring about $5 \times 4 \mathrm{~cm}$ (Figure 1). The mass was firm to hard in consistency and mildly tender. There was no active bleeding. Plain skull X-ray (posterior-anterior (PA) and lateral views) showed a well-organized soft tissue mass in the left paranasal region with no bony fracture. Differential diagnoses considered were hemophilic pseudotumor, ameloblastoma and odontogenic cysts.

Coagulation Screen tests results showed a prothrombin time (PT) of 16 seconds (control 14 secs) and prolonged activated partial thromboplastin time (aPTT) of 138 seconds (control 36 secs). Mixing study done was suggestive of Hemophilia A. with an estimated factor VIII level of $3 \%$, that is a moderate form of the disease. A diagnosis of left paranasal hemophilic pseudotumor was made.

He was then commenced on factor VIII concentrate (Eloctate) at $25 \mathrm{U} / \mathrm{kg}$ daily for 1 week, then 3 times weekly for the next four weeks. There was a complete resolution of the para-nasal mass at five (5) weeks post trauma (Figure 2). 


\section{Discussion}

Hemophilic pseudotumors are complications of Hemophilia occurring most commonly among people with severe disease. Our case is a moderate form of the disease with a factor level of $3 \%$. Pseudotumors affect $1-2 \%$ of patients with severe disease and usually occurs in the bone due to either repeated untreated or poorly treated bleeding. ${ }^{1}$ Clinically, they present as progressive painless swellings over a long period of time. ${ }^{2}$ Hemophilic pseudotumors, depending on the site of occurrence, can affect the soft tissue, subperiostium or the bone cortex. ${ }^{3}$ Soft tissue involvement is seen in about $14 \%$ of cases while affectation of the bone is seen in $86 \%{ }^{4}$ They are have been classified into either proximal or distal pseudotumours. ${ }^{5}$ Proximal pseudotumors are those mainly occurring in the long bones (especially the femur) and the pelvis of the mature skeleton of adult hemophilic patients and are usually associated with trauma and recurrent bleeding, more especially into muscles adjacent to the bone. They usually develop over years. Distal pseudotumors are those that mostly occur in the young patient with open epiphyseal growth plates, commonly affecting the small bones of hands and feet of the immature skeleton and are of intraosseous origin. The distal pseudotumors develop rapidly and are not commonly associated with direct trauma. Generally, prior history of trauma is seen in about $57 \%$ of cases and this pattern is

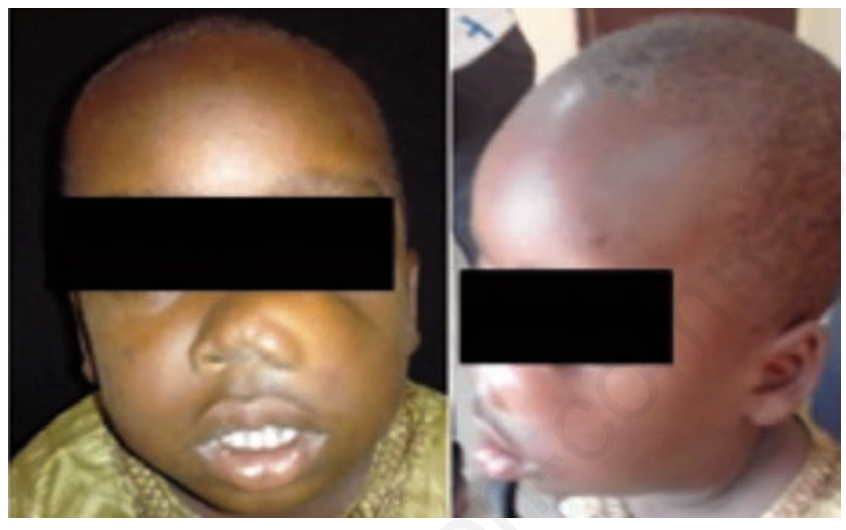

Figure 1. Left paranasal pseudotumor: two weeks post-trauma.

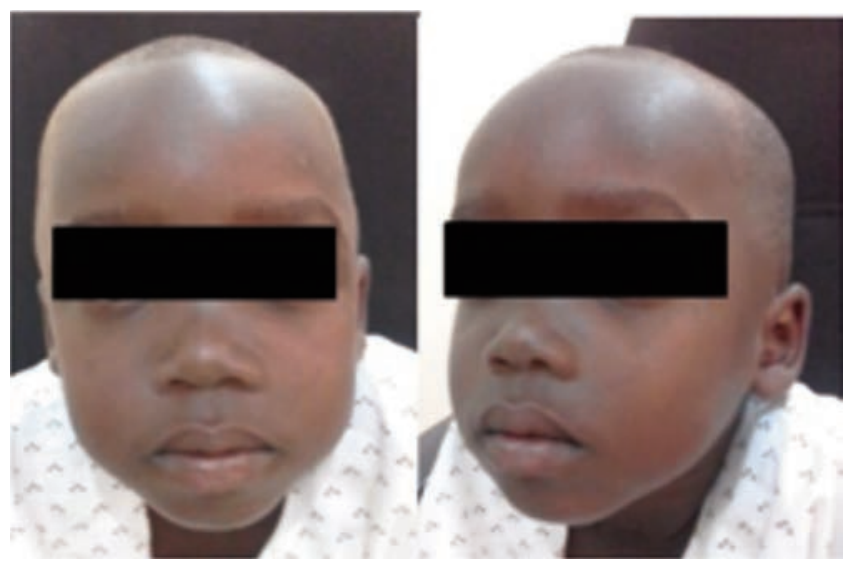

Figure 2. Complete resolution of the swelling five weeks post-trauma. what was seen in our case report. ${ }^{4}$

Radio-imaging techniques such as Computed Tomography (CT), Magnetic Resonance Imaging and X-rays are very important in the diagnosis of pseudotumors. However, those occurring in the soft tissues are very difficult to diagnose using plain X-rays. Magnetic resonant imaging (MRI) and CT scans are very good in the characterization of soft tissue pseudotumours. ${ }^{6}$

The management of pseudotumors can either be conservative or by surgical intervention. ${ }^{7}$ Conservative management includes the use of factor replacement therapy, embolization as well as some local measures such as immobilization and in some cases radiotherapy. Conservative care is very effective in the care of small to intermediate sized pseudotumors in the absence of other complications such as pressure effects with neuromuscular deficits and increased risk of rupture. Surgical interventions are indicated for large lesions, soft-tissue pseudotumors when rupture is imminent, presence of neuromuscular deficits or failure of conservative therapy. ${ }^{8}$ Our patient who is 5 years old developed the pseudotumor following trauma. This is consistent with the etiology in most of the patients with hemophilic pseudotumours. ${ }^{4}$ The diagnosis was made using clinical evaluation, coagulation studies and plain X-ray. He did well with conservative care with factor VIII replacement therapy. The target for the therapy was $50 \%$ factor level. This was achieved by giving factor VIII concentrate at the dose of $25 \mathrm{IU} / \mathrm{kg}$. Surgical intervention was avoided in this patient because there was no evidence of respiratory compromise. We observed complete resolution of the swelling at the end of 5 weeks of factor replacement.

\section{Conclusions}

A high index of suspicion, careful history taking and assessment of all patients presenting with swellings may help to unmask hemophilic pseudotumors, as well as prevent bleeding complications that may arise following biopsies or surgical intervention(s) without adequate clotting factor coverage.

\section{References}

1. Faisal YS, Faizan YS, Shahid B. Hemophilicpseudotumors: Natural history and a case report of pseudotumor with femur fracture and swan-neck deformity. Int J Orthop Sci 2016;2:104.

2. Panotopoulos J, Ay C, Trieb K, et al. Surgical treatment of the hemophilic pseudotumor: A single centre experience. Int Orthop 2012:2157-62.

3. Gupta S, Mohapatra BB, Ghai S, et al. Hemophilic pseudotumor of the paranasal sinuses: management with radiotherapy and factor replacement therapy. Haemophilia 2001;7:595-9.

4. Xue F, Sun C, Sui T, et al. Hemophilic Pseudotumor in Chinese Patients: A Retrospective Single-Centered Analysis of 14 Cases. Clin Appl Thromb Hemost 2011;279-82.

5. Gilbert MS. The hemophilic pseudotumor. Prog Clin Biol Res 1990;324:257-62.

6. Park JS, Ryu KN. Hemophilicpseudotumor involving the musculoskeletal system: spectrum of radiologic findings. AJR Am J Roentgenol 2004;183:55-61.

7. Cox DP, Solar A, Huang J, Chigurupati R. Pseudotumor of the Mandible as First Presentation of Hemophilia in a 2-Year-Old Male: A Case Report and Review of Jaw Pseudotumors of Hemophilia. Head Neck Pathol 2011;5:226-32. 